Case report

\title{
Automatic implantable cardioverter defibrillator pocket infection due to Providencia rettgeri: a case report Jorge Manuel Marull ${ }^{1 *}$ and Maria Elena De Benedetti ${ }^{2}$
}

\author{
Addresses: ${ }^{1}$ Department of Internal Medicine, St Luke's-Roosevelt Hospital Center-Columbia University College of Physicians and Surgeons, \\ $100010^{\text {th }}$ Avenue, New York, NY, 10019, USA \\ ${ }^{2}$ Department of Internal Medicine, St Luke's-Roosevelt Hospital Center-Columbia University College of Physicians and Surgeons, $100010^{\text {th }}$ \\ Avenue, New York, NY, 10019, USA \\ Email: JMM* - jmarull@chpnet.org; MEDB - mdbzunino@chpnet.org \\ * Corresponding author \\ Received: 28 January 2009 Accepted: 20 June 2009 Published: 24 August 2009 \\ Cases Journal 2009, 2:8607 doi: 10.4076/1757-1626-2-8607 \\ This article is available from: http://casesjournal.com/casesjournal/article/view/8607 \\ (c) 2009 Marull and De Benedetti; licensee Cases Network Ltd. \\ This is an Open Access article distributed under the terms of the Creative Commons Attribution License (http://creativecommons.org/licenses/by/3.0), \\ which permits unrestricted use, distribution, and reproduction in any medium, provided the original work is properly cited.
}

\begin{abstract}
Coagulase-negative staphylococci and Staphylococcus aureus are the commonest pathogens involved in infections of pacemaker-defibrillator systems. Among causative Gram-negative bacteria, infections due to Klebsiella, Serratia, Pseudomonas, Acinetobacter and other species have been reported. We report herein a unique case of an automatic implantable cardioverter defibrillator infection due to Providencia rettgeri in a 65-year-old male who was admitted to our service with bacteremia and infection of the generator and subcutaneous array in a recently implanted device.
\end{abstract}

\section{Introduction}

Insertion of an automatic implantable cardioverter-defibrillator (ICD) reduces the occurrence of sudden death in patients at risk for ventricular tachycardia or ventricular fibrillation [1]. Despite the advent of transvenously placed devices and improvements in surgical techniques, device infection is still a serious complication due to associated morbidity, mortality and financial costs [2]. Management of this clinical entity is challenging since no current guidelines are available for optimal treatment. We present a case of an ICD pocket infection due to an uncommon pathogen.

\section{Case presentation}

This is a case of a 65-year-old Jewish male who presented to our hospital complaining of a two-week history of serosanguineous oozing coming from a healing surgical wound overlying an ICD that had been implanted sixteen days prior to the day of admission at a different medical center. No purulent discharge was seen. The area became increasingly painful. The patient denied any fevers, chills, palpitations or other cardiovascular symptoms on admission. Vital signs were as follows: Temperature of $98.4^{\circ} \mathrm{F}$, Blood pressure of 105/64 mmHg, Heart rate of 60 beats per minute, Respiratory rate of 14 breaths per minute and Oxygen saturation of $98 \%$ on room air. Physical examination revealed an eight-centimeter irregularly oval, warm, swollen, erythematous and tender area on the left anterior-superior chest wall. No local ulcers or new heart murmurs were noticed. Poor dentition, together with the presence of disheveling appearance was appreciated.

Past medical history was positive for non-ischemic dilated cardiomyopathy, congestive heart failure (NYHA class III), atrial fibrillation, essential hypertension and a recent 
Table I. Hematologic and blood chemistry laboratory data

\begin{tabular}{|c|c|c|c|c|c|}
\hline \multirow{2}{*}{$\begin{array}{l}\text { Hematology } \\
\text { White cell count }\end{array}$} & \multicolumn{5}{|c|}{ Chemistry } \\
\hline & 10.5 & Sodium & 138 & Protein & 6.2 \\
\hline Hemoglobin & $|2|$. & Potassium & 4.6 & Albumin & 3.3 \\
\hline Hematocrit & 34.8 & Chloride & 105 & Total Bilirrubin & 0.5 \\
\hline Platelet & 231 & BUN & 23 & AST & 40 \\
\hline PTT & 56 & Creatinine & 1.2 & ALT & 49 \\
\hline Prothromin time & 29.7 & Glucose & 89 & Alk Phosp & 43 \\
\hline INR & 2.9 & C Reactive protein & 1.6 & & \\
\hline
\end{tabular}

Ref: Partial thromboplastin time (PTT), International Normal Ratio (INR), Blood Urea Nitrogen (BUN), Aspartate aminotransferase (AST), Alanine aminotransferase (ALT), Alkaline Phosphatase (Alk Phos).

syncopal episode. The patient had no history of alcohol, tobacco or illegal drug abuse. He was a retired messenger since 2004 and lived in a shelter. No family history of cardiomyopathy or oncologic diseases was noted.

Admission laboratory findings revealed mild leukocytosis together with mild normocytic normochromic anemia (Table 1). An electrocardiogram showed atrial paced rhythm at 60 beats per minute, a QRS complex of 102 msec and a QTc interval of $480 \mathrm{sec}$, no signs of ischemia were recorded. His home medications included Warfarin, Carvedilol, Lisinopril and Simvastatin.

Providencia rettgeri grew in two sets of deep wound cultures, one set of superficial wound cultures, one set of peripheral blood cultures obtained on the day of admission as well as from the culture of the removed device. The antibiotic resistance pattern was similar in all the cultures (Table 2). A trans-esophageal echocardiography did not reveal vegetations or signs of endocarditis.

The entire implanted system, including the cardiac leads of a dual chamber cardioverter-defibrillator, Medtronic ${ }^{\circledR}$, model D224DRG, Secura DR was removed on day two of admission. The wound was left opened, and dry to wet dressings were applied locally. Resolution of the infection was seen after the patient received a four-week course of intravenous Cefepime two grams every twelve hours, subsequent surveillance blood cultures revealed no growth and second intention healing of the surgical wound ensued. An external defibrillator vest was initially placed and as soon as the antibiotic regimen was completed a new dual chamber cardioverter-defibrillator was placed on the right side of the thoracic wall. The patient was subsequently discharged with adequate follow-up.

\section{Discussion}

Providencia rettgeri (PR) has been frequently associated with colonization of indwelling urinary catheters and urinary tract infections, bacteremias, skin infections, traveler's diarrhea, etc $[3,4]$. To the best of our knowledge, this microorganism has never been implicated in infections of automatic ICD, being this case the first one ever reported in the literature as evidenced by a MEDLINE database search. Axillae, human feces, urine, throat and perineum could serve as potential reservoirs for Providencia species, mainly in chronic debilitated patients. Exogenous sources include sewage contaminated natural waters [5]. A probable key feature in the pathogenesis of this patient's infection was the formation of a biofilm composed mainly of host adhesins (fibrinogen, fibronectin, collagen) and bacteria that may have occurred soon after the insertion of the ICD [6]. The skin of our patient could have been colonized with PR before the adherence of the bacterium to the device since no foley catheter insertion had been reported on prior admissions.

PR is a facultative anaerobe, Gram-negative, motile, opportunistic bacterium that belongs to the genus Providencia, tribe Proteeae (along with the genus Proteus and Morganella), Enterobacteriaceae family. PR was first isolated by Rettger in 1904 during an epidemic of fowl cholera, but it was only studied in detail later in 1918 when

Table 2. Resistance profile of organism isolate

\begin{tabular}{|c|c|c|c|c|c|}
\hline Amikacin & $<=16$ & $S$ & Ciprofloxacin & $<=1$ & $\mathrm{~S}$ \\
\hline Amoxicillin/clavulanate & $>16 / 8$ & $\mathrm{R}$ & Gentamicin & $<=4$ & $\mathrm{~S}$ \\
\hline Ampicillin & $>16$ & $\mathrm{R}$ & Imipenem & $<=4$ & $\mathrm{~S}$ \\
\hline Ampicillin/sulbactam & $16 / 8$ & $\mathrm{I}$ & Levofloxacin & $<=2$ & $\mathrm{~S}$ \\
\hline Cefazolin & $>16$ & $\mathrm{R}$ & Moxifloxacin & $<=2$ & $\mathrm{~S}$ \\
\hline Cefepime & 30 & $\mathrm{~S}$ & Piperacillin/tazobactam & $<=16$ & $\mathrm{~S}$ \\
\hline Cefoxitin & $<=8$ & $\mathrm{~S}$ & Ticarcillin/clavulanate & $<=16$ & $\mathrm{~S}$ \\
\hline Ceftazidime & $<=8$ & $\mathrm{~S}$ & Tobramycin & $<=4$ & $\mathrm{~S}$ \\
\hline Ceftriaxone & $<=8$ & $\mathrm{~S}$ & Trimethoprim/Sulfa & $>2 / 38$ & $\mathrm{R}$ \\
\hline
\end{tabular}

Ref: Susceptible (S), Intermediate (I), Resistant (R). 
it was called Bacterium rettgerei [7]. In 1951, Kauffmann applied the term Providencia to the genus described by Stuart et al. at Brown University in Providence, Rhode Island [8]. Many taxonomic changes of genus Providencia have taken place since then, which included a frequent overlap between the Gram-negative genera belonging to the tribe Proteeae. Currently, the genus Providencia includes five species: $P$. stuartii, $P$. rettgeri, $P$. alcalifacens, $P$. rustigianii and $P$. heimbachae. Providencia stuartii has been described as the commonest isolate in residents of nursing homes diagnosed with bacteremia [9]. In a recent epidemiologic study in a Canadian population, the incidence of isolates for the genus Providencia was found to be 3.4/100 000/year, with more infections reported as people aged and were residing in nursing homes [10].

Nosocomial outbreaks of highly resistant strains of PR causing urinary tract infections and bacteremias have been described. Epidemiologic factors included the intensive concomitant use of antibiotics and indwelling urinary catheters; while the use of gloves by personnel showed to be an effective control measure $[11,12]$. Our patient was kept on contact isolation until the end of the admission with no further cases reported on the same medical ward.

Resistant Enterobacteriaceae is a recent major health hazard. The transmission of resistance is frequently acquired through plasmids from other Gram-negative rods and it has been associated with the increasing use of antibiotics. The frequent isolation of extended spectrum beta-lactamase in Providencia species alarms about the risk of future nosocomial epidemics caused by this multiresistant organism and is a considered an emerging problem $[13,14]$. The natural antibiotic susceptibility of PR includes numerous beta-lactam, fluoroquinolone and aminoglycoside antibiotics and a less resistant pattern than that of $P$. stuartii [15].

\section{Conclusion}

Infections by PR and other Gram-negative bacteria should be considered when approaching a recent hospitalized patient with an early automatic ICD infection, and initial empiric antibiotic coverage for this bacterium may be warranted. The election of the antibiotic should be guided by culture susceptibilities given the increasing evidence of multi-drug resistant strains.

\section{Abbreviations}

ICD, Automatic implantable cardioverter-defibrillator; NYHA, New York Heart Association; PR, Providencia rettgeri; P. stuartii, Providencia stuartii.

\section{Consent}

The following case was evaluated and approved by the Institutional Review Board (IRB). Written informed consent could not be obtained because the patient was lost to follow-up. Despite repeated attempts we were unable to contact the patient. Every effort has been made to keep the patient's identity anonymous. We would not expect the patient to object to publication.

\section{Competing interests}

The authors declare that they have no competing interests.

\section{Authors' contributions}

JM was a major contributor in writing the manuscript, analyzing the data, and collecting the information in the inpatient setting. $M D$ wrote the abstract and made a contribution in the conclusion. Both authors read and approved the final manuscript.

\section{References}

I. Winkle RA, Mead RH, Ruder MA, Gaudiani VA, Smith NA, Buch WS, Schmidt $P$, Shipman T: Long-term outcome with the automatic implantable cardioverter-defibrillator. J Am Coll Cardiol 1989, I3:|353-1361.

2. Lai KK, Fontecchio DA: Infections associated with implantable cardioverter defibrillators placed transvenously and via thoracotomies: epidemiology, infection control, and management. Clin Infect Dis 1998, 27:265-269.

3. Russo TA, Johnson JR: Diseases caused by Gram-negative enteric bacilli. In Harrison's Principles of Internal Medicine Volume I. 17th edition. Edited by Braunwald E, Fauci AS, Kasper DL, Hauser SL. New York: McGraw-Hill; 2008:937-944.

4. Yoh M, Matsuyama J, Ohnishi M, Takagi K, Miyagi H, Mori K, Park KS, Ono T, Honda T: Importance of Providencia species as a major cause of travellers' diarrhoea. J Med Microbiol 2005, 54:1077-1082.

5. Stickler DJ, Fawcett C, Chawla JC: Providencia stuartii: A search for its natural habitat. J Hosp Infect 1985, 6:221-223.

6. Darouiche RO: Treatment of Infections Associated with Surgical Implants. N Engl J Med 2004, 350: |422- | 429.

7. Hadley PB, Elkins MW, Caldwell DW: The colon-typhoid intermediates as causative agents of disease in birds. I. The paratyphoid bacteria. $R$ I Agric Exp Stn Bull 1918, I74:1-216.

8. Kauffmann F: Enterobacteriaceae. Copenhagen: Einar Munksgaard; I95I.

9. Muder RR, Brennen C, Wagener MM, Goetz AM: Bacteremia in a long-term-care facility: a five-year prospective study of 163 consecutive episodes. Clin Infect Dis 1992, 14:647-654.

10. Laupland KB, Parkins MD, Ross T, Pitout JD: Population-based laboratory surveillance for tribe Proteeae isolates in a large Canadian health region. Clin Microbiol Infect 2007, I 3:683-688.

II. Kaslow RA, Lindsey JO, Bisno AL, Price A: Nosocomial infection with highly resistant Proteus rettgeri. Report of an epidemic. Am J Epidemiol 1976, I04:278-286.

12. Edwards LD, Cross A, Levin S, Landau W: Outbreak of a nosocomial infection with a strain of Proteus rettgeri resistant to many antimicrobials. Am J Clin Pathol I 974, 6 I:4 I-46.

13. Shiroto K, Ishii Y, Kimura S, Alba J, Watanabe K, Matsushima Y, Yamaguchi K: Metallo-beta-lactamase IMP-I in Providencia rettgeri from two different hospitals in Japan. J Med Microbiol 2005, 54:1065-1070.

14. Tumbarello M, Citton R, Spanu T, Sanguinetti M, Romano L, Fadda G, Cauda R: ESBL-producing multidrug-resistant Providencia stuartii infections in a university hospital. J Antimicrob Chemother 2004, 53:277-282.

15. Stock I, Wiedemann B: Natural antibiotic susceptibility of Providencia stuartii, $\mathbf{P}$. rettgeri, $\mathbf{P}$. alcalifaciens and $\mathbf{P}$. rustigianii strains. J Med Microbiol 1998, 47:629-642. 